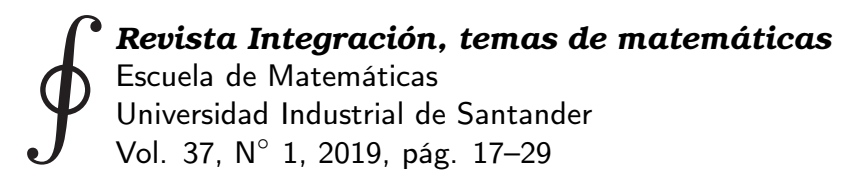

DOI: http://dx.doi.org/10.18273/revint.v37n1-2019002

\title{
The property of Kelley and continua
}

\author{
Sergio MaCías* \\ Universidad Nacional Autónoma de México, Instituto de Matemáticas, México D.F., \\ México.
}

\begin{abstract}
We study Hausdorff continua with the property of Kelley. We present Hausdorff version of several results known in the metric case. We also establish a weak Hausdorff version of Jones' Aposyndetic Decomposition Theorem.

Keywords: Atomic map, Effros continuum, homogeneous continuum, property of Kelley, set function $\mathcal{T}$, uniform property of Effros.

MSC2010: 54B20, 54C60.
\end{abstract}

\section{La propiedad de Kelley y continuos}

Resumen. Estudiamos a los continuos de Hausdorff con la propiedad de Kelley. Presentamos la versión para continuos de Hausdorff de varios resultados conocidos en el caso métrico. Establecemos una versión débil de Hausdorff del Teorema de Descomposición Aposindética de Jones.

Palabras clave: Continuo de Effros, continuo homogéneo, función atómica, función $\mathcal{T}$, propiedad uniforme de Effros, propiedad de Kelley.

\section{Introduction}

The first person to investigate contractibility of hyperspaces was M. Wojdysławski [20] in 1938. The next investigation was done by J. L. Kelley [7] in 1942. Kelley defined a property as a sufficient condition to guarantee the contractibility of some of the hyperspaces of metric continua. This property has been known as property 3.2, property $[\kappa]$ and property of Kelley. It turns out that this property has been very important in the study not only of hyperspaces, but of metric continua, too [18, Chapter XVI]. In 1999 W. J. Charatonik [3] and W. Makuchowski [14] defined the property of Kelley for Hausdorff continua. Charatonik presented a homogeneous Hausdorff continuum without the property of Kelley, and Makuchowski generalized some local connectedness properties to hyperspaces of Hausdorff continua. We present Hausdorff version of several results known in the metric case. We also establish a weak Hausdorff version of Jones' Aposyndetic Decomposition Theorem.

\footnotetext{
* Email: sergiom@matem. unam.mx

Received: 22 August 2018, Accepted: 17 October 2018.

To cite this article: S. Macías, The property of Kelley and continua, Rev. Integr. temas mat. 37 (2019), No. 1, 17-29. doi: 10.18273/revint.v37n1-2019002.
} 


\section{Definitions}

If $Z$ is a topological space, then given a subset $A$ of $Z$, the interior of $A$ is denoted by $\operatorname{Int}(A)$ and the closure of $A$ by $C l(A)$. We write $\operatorname{Int}_{Z}(A)$ and $C l_{Z}(A)$, respectively, if there is a possibility of confusion.

A map is a continuous function. A surjective map $f: X \rightarrow Y$ between topological spaces is monotone provided that $f^{-1}(C)$ is connected for every connected subset $C$ of $Y$. The surjective map $f$ is open (closed, respectively) if $f(U)$ is open (closed, respectively) in $Y$ for each open (closed, respectively) set $U$ of $X$. If $f: X \rightarrow Y$ is a surjective map and $Z$ is a nonempty subset of $X$, then $\left.f\right|_{Z}: Z \rightarrow Y$ denotes the restriction of $f$ to $Z$. Given a space $Z, 1_{Z}$ denotes the identity map on $Z$.

Given a topological space $Z$, a decomposition of $Z$ is a family $\mathcal{G}$ of nonempty and mutually disjoint subsets of $Z$ such that $\bigcup \mathcal{G}=Z$. A decomposition $\mathcal{G}$ of a topological space $Z$ is said to be upper semicontinuous if for each element $G$ of $\mathcal{G}$ and each open subset $U$ of $Z$ such that $G \subset U$, there exists an open subset $V$ of $Z$ such that $G \subset V$, and for every $G^{\prime} \in \mathcal{G}$ such that $G^{\prime} \cap V \neq \emptyset$, we have that $G^{\prime} \subset U$. The decomposition $\mathcal{G}$ of $Z$ is lower semicontinuous provided that for each $G \in \mathcal{G}$ any two points $z_{1}$ and $z_{2}$ of $G$ and any open subset $U$ of $Z$ containing $z_{1}$, there exists an open subset $V$ of $Z$ such that $z_{2} \in V$ and for each $G^{\prime} \in \mathcal{G}$ such that $G^{\prime} \cap V \neq \emptyset$, we obtain that $G^{\prime} \cap U \neq \emptyset$. The decomposition $\mathcal{G}$ is continuous if it is both upper and lower semicontinuous.

A continuum is a compact connected Hausdorff space. A subcontinuum of a space $Z$ is a continuum contained in $Z$. A continuum is decomposable if it is the union of two of its proper subcontinua. A continuum is indecomposable if it is not decomposable. A continuum $X$ is homogeneous provided that for each pair of points $x_{1}$ and $x_{2}$ of $X$, there exists a homeomorphism $h: X \rightarrow X$ such that $h\left(x_{1}\right)=x_{2}$.

Given a continuum $X$, we define the set function $\mathcal{T}$ as follows: if $A$ is a subset of $X$, then

$$
\begin{gathered}
\mathcal{T}(A)=X \backslash\{x \in X \mid \text { there exists a subcontinuum } W \text { of } X \text { such that } \\
\qquad x \in \operatorname{Int}(W) \subset W \subset X \backslash A\} .
\end{gathered}
$$

We write $\mathcal{T}_{X}$ if there is a possibility of confusion. Let us observe that for any subset $A$ of $X, \mathcal{T}(A)$ is a closed subset of $X$ and $A \subset \mathcal{T}(A)$. A continuum $X$ is aposyndetic provided that $\mathcal{T}(\{p\})=\{p\}$ for every $p \in X$.

The set function $\mathcal{T}$ is idempotent on $X$ (idempotent on closed sets of $X$, on subcontinua of $X$, respectively) provided that $\mathcal{T}^{2}(A)=\mathcal{T}(A)$ for each subset $A$ of $X$ (for every nonempty closed subset $A$ of $X$, for each subcontinuum $A$ of $X$, respectively), where $\mathcal{T}^{2}=\mathcal{T} \circ \mathcal{T}$. The set function $\mathcal{T}$ is idempotent on singletons if $\mathcal{T}^{2}(\{x\})=\mathcal{T}(\{x\})$ for all $x \in X$.

We say that $\mathcal{T}$ is continuous for a continuum $X$ provided that $\mathcal{T}: 2^{X} \rightarrow 2^{X}$ is continuous, where $2^{X}$ is the hyperspace of nonempty closed subsets of $X$, topologized with the Vietoris topology [15] (or the Hausdorff metric if $X$ is metric [18]). A base for the Vietoris topology for $2^{X}$ is given by the sets of the form

$$
\left\langle U_{1}, \ldots, U_{m}\right\rangle=\left\{A \in 2^{X} \mid A \subset \bigcup_{j=1}^{m} U_{j} \text { and } A \cap U_{j} \neq \emptyset \text { for all } j\right\},
$$

[Revista Integración, temas de matemáticas 
where $U_{1}, \ldots, U_{m}$ are open subsets of $X$. If $f: X \rightarrow Y$ is a map, then $2^{f}: 2^{X} \rightarrow 2^{Y}$ given by $2^{f}(A)=f(A)$ is a map $[18,(1.168)]$. Observe that there exists a copy of $X$ inside $2^{X}$, namely, $\mathcal{F}_{1}(X)=\{\{x\} \mid x \in X\}$. The map $\xi: X \rightarrow \mathcal{F}_{1}(X)$ given by $\xi(x)=\{x\}$ is an embedding. We say that $\mathcal{T}$ is continuous on singletons provided that $\left.\mathcal{T}\right|_{\mathcal{F}_{1}(X)}: \mathcal{F}_{1}(X) \rightarrow 2^{X}$ is continuous. For more information about the set function $\mathcal{T}$ see [8, Chapter 3]

Let $X$ be a continuum. Then $\mathcal{C}_{1}(X)$ denotes the hyperspace of subcontinua of $X$; i.e.,

$$
\mathcal{C}_{1}(X)=\left\{A \in 2^{X} \mid A \text { is connected }\right\} .
$$

If $U_{1}, \ldots, U_{m}$ are open subsets of $X$, then $\left\langle U_{1}, \ldots, U_{m}\right\rangle \cap \mathcal{C}_{1}(X)$ is denoted by $\left\langle U_{1}, \ldots, U_{m}\right\rangle_{1}$. Note that if $K$ is a subcontinuum of $X$, then $\mathcal{T}(K)$ is a subcontinuum of $X$ (Theorem 3.5).

\section{Preliminaries}

The following result is known as The Boundary Bumping Theorem. It has many applications in continuum theory and in hyperspaces. A proof of this theorem may be found in [6, Theorem 2-16]. $B d(U)$ denotes the boundary of a set $U$ in a space $X$.

Theorem 3.1. Let $X$ be a continuum and let $U$ be a nonempty, proper, open subset of $X$. If $K$ is a component of $C l(U)$, then $K \cap B d(U) \neq \emptyset$.

Let $X$ be a continuum and let $K$ be a subcontinuum of $X$. Then $K$ is a terminal subcontinuum of $X$ if for each subcontinuum $L$ of $X$ such that $L \cap K \neq \emptyset$, then either $L \subset K$ or $K \subset L$.

Lemma 3.2. Let $X$ be a continuum. If $W$ is a proper terminal subcontinuum of $X$, then $\operatorname{Int}(W)=\emptyset$.

Proof. Suppose $W$ is a proper terminal subcontinuum of $X$ and $\operatorname{Int}_{X}(W) \neq \emptyset$. Note that $B d_{X}\left(\operatorname{Int}_{X}(W)\right) \subset W$. Let $x \in X \backslash W$ and let $C$ be the component of $X \backslash \operatorname{Int}_{X}(W)$ containing $x$. By Theorem 3.1, $C \cap B d_{X}\left(\operatorname{Int}_{X}(W)\right) \neq \emptyset$. Hence, $C \cap W \neq \emptyset$, and $C \backslash W \neq \emptyset$. Since $W$ is a terminal subcontinuum of $X, W \subset C$, a contradiction to the fact that $C \cap \operatorname{Int}_{X}(W)=\emptyset$. Therefore, $\operatorname{Int}_{X}(W)=\emptyset$.

Lemma 3.3. If $X$ is a continuum, then $\mathcal{T}$ is upper semicontinuous.

Proof. Let $U$ be an open subset of $X$ and let $\mathcal{U}=\left\{A \in 2^{X} \mid \mathcal{T}(A) \subset U\right\}$. We show that $\mathcal{U}$ is open in $2^{X}$. Let $B \in C l_{2^{X}}\left(2^{X} \backslash \mathcal{U}\right)$. Then there exists a net $\left\{B_{\lambda}\right\}_{\lambda \in \Lambda}$ of elements of $2^{X} \backslash \mathcal{U}$ converging to $B[5,1.6 .3]$. Note that for each $\lambda \in \Lambda, \mathcal{T}\left(B_{\lambda}\right) \cap(X \backslash U) \neq \emptyset$. Let $x_{\lambda} \in \mathcal{T}\left(B_{\lambda}\right) \cap(X \backslash U)$. Without loss of generality, we assume that $\left\{x_{\lambda}\right\}_{\lambda \in \Lambda}$ converges to a point $x \in X[5,3.1 .23$ and 1.6.1] and [17, Theorem 4]. Note that $x \in X \backslash U$. We assert that $x \in \mathcal{T}(B)$. Suppose $x \in X \backslash \mathcal{T}(B)$. Then there exists a subcontinuum $W$ of $X$ such that $x \in \operatorname{Int}(W) \subset W \subset X \backslash B$. Since $\left\{B_{\lambda}\right\}_{\lambda \in \Lambda}$ converges to $B$ and $\left\{x_{\lambda}\right\}_{\lambda \in \Lambda}$ converges to $x$, there exists $\lambda_{0} \in \Lambda$ such that for each $\lambda \geq \lambda_{0}, B_{\lambda} \subset X \backslash W$ and $x_{\lambda} \in \operatorname{Int}(W)$.

Vol. 37, $\left.\mathrm{N}^{\circ} 1,2019\right]$ 
Let $\lambda \geq \lambda_{0}$. Then $x \in \operatorname{Int}(W) \subset W \subset X \backslash B_{\lambda}$. This implies that $x_{\lambda} \in X \backslash \mathcal{T}\left(B_{\lambda}\right)$, a contradiction. Thus, $x \in \mathcal{T}(B) \cap(X \backslash U)$. Hence, $B \in 2^{X} \backslash \mathcal{U}$. Therefore, $2^{X} \backslash \mathcal{U}$ is closed in $2^{X}$ and $\mathcal{U}$ is open.

Lemma 3.4. Let $X$ and $Y$ be continua and let $h: X \rightarrow Y$ be a homeomorphism. If $A$ is a subset of $X$, then $h\left(\mathcal{T}_{X}(A)\right)=\mathcal{T}_{Y}(h(A))$.

Proof. Let $A$ be a closed subset of $X$. Let $y \in Y \backslash h\left(\mathcal{T}_{X}(A)\right)$. Then $h^{-1}(y) \in X \backslash \mathcal{T}_{X}(A)$. Hence, there exists a subcontinuum $W$ of $X$ such that $h^{-1}(y) \in \operatorname{Int}(W) \subset W \subset X \backslash A$. This implies that $y \in \operatorname{Int}(h(W)) \subset h(W) \subset Y \backslash h(A)$. Thus, $y \in Y \backslash \mathcal{T}_{Y}(h(A))$.

Now, let $y \in X \backslash \mathcal{T}_{Y}(h(A))$. Then there exists a subcontinuum $K$ of $Y$ such that $y \in \operatorname{Int}(K) \subset K \subset Y \backslash h(A)$. This implies that $h^{-1}(y) \in \operatorname{Int}\left(h^{-1}(K)\right) \subset h^{-1}(K) \subset X \backslash A$. Thus, $h^{-1}(y) \in X \backslash \mathcal{T}_{X}(A)$. Hence, $y \in Y \backslash h(\mathcal{T}(A))$.

Therefore, $h(\mathcal{T}(A))=\mathcal{T}(h(A))$.

Theorem 3.5. Let $X$ be a continuum. If $W$ is a subcontinuum of $X$, then $\mathcal{T}(W)$ is a subcontinuum of $X$.

Proof. Let $W$ be a subcontinuum of $X$. We already know that $\mathcal{T}(W)$ is a closed subset of $X$.

Suppose $\mathcal{T}(W)$ is not connected. Then there exist two disjoint closed subsets $A$ and $B$ of $X$ such that $\mathcal{T}(W)=A \cup B$. Since $W$ is connected, without loss of generality, we assume that $W \subset A$. Since $X$ is a normal space, there exists an open subset $U$ of $X$ such that $A \subset U$ and $C l(U) \cap B=\emptyset$.

Note that $B d(U) \cap \mathcal{T}(W)=\emptyset$. Hence, for each $z \in B d(U)$, there exists a subcontinuum $K_{z}$ of $X$ such that $z \in \operatorname{Int}\left(K_{z}\right) \subset K_{z} \subset X \backslash W$. Since $B d(U)$ is compact, there exist $z_{1}, \ldots, z_{n} \in B d(U)$ such that $B d(U) \subset \bigcup_{j=1}^{n} \operatorname{Int}\left(K_{z_{j}}\right) \subset \bigcup_{j=1}^{n} K_{z_{j}}$. Let $V=U \backslash\left(\bigcup_{j=1}^{n} K_{z_{j}}\right)$. Let $Y=X \backslash V=(X \backslash U) \bigcup\left(\bigcup_{j=1}^{n} K_{z_{j}}\right)$. By Theorem 3.1, $Y$ has only a finite number of components. Observe that $B \subset X \backslash C l(U) \subset X \backslash U \subset Y$. Hence, $B \subset \operatorname{Int}(Y)$. Let $b \in B$, and let $C$ be the component of $Y$ such that $b \in C$. Note that $b \in \operatorname{Int}(C)$. By construction, $C \cap W=\emptyset$. Thus, $b \in X \backslash \mathcal{T}(W)$, a contradiction. Therefore, $\mathcal{T}(W)$ is connected.

Let $X$ be a continuum and let $z \in X$. We say that $\mathcal{T}(\{z\})$ has property $B L[9$, p. 167] provided that $\mathcal{T}(\{z\}) \subset \mathcal{T}(\{x\})$ for each $x \in \mathcal{T}(\{z\})$.

Lemma 3.6. Let $X$ be a decomposable continuum for which $\mathcal{T}$ is idempotent on singletons, and let $z \in X$. If $\mathcal{T}(\{z\})$ has property $B L$, then $\mathcal{T}(\{w\})=\mathcal{T}(\{z\})$ for every $w \in \mathcal{T}(\{z\})$.

Proof. Let $z \in X$ be such that $\mathcal{T}(\{z\})$ has property $B L$, and let $w \in \mathcal{T}(\{z\})$. Since $w \in \mathcal{T}(\{z\})$ and $\mathcal{T}$ is idempotent on singletons, $\mathcal{T}(\{w\}) \subset \mathcal{T}(\mathcal{T}(\{z\}))=\mathcal{T}(\{z\})$. Hence, $\mathcal{T}(\{w\}) \subset \mathcal{T}(\{z\}) . \quad$ Since $\mathcal{T}(\{z\})$ has property $B L, \mathcal{T}(\{z\}) \subset \mathcal{T}(\{w\})$. Therefore, $\mathcal{T}(\{w\})=\mathcal{T}(\{z\})$. 
Corollary 3.7. Let $X$ be a decomposable continuum for which $\mathcal{T}$ is idempotent on singletons. If $z_{1}$ and $z_{2}$ are two points of $X$ such that both $\mathcal{T}\left(\left\{z_{1}\right\}\right)$ and $\mathcal{T}\left(\left\{z_{2}\right\}\right)$ have property $B L$, then either $\mathcal{T}\left(\left\{z_{1}\right\}\right)=\mathcal{T}\left(\left\{z_{2}\right\}\right)$ or $\mathcal{T}\left(\left\{z_{1}\right\}\right) \cap \mathcal{T}\left(\left\{z_{2}\right\}\right)=\emptyset$.

Proof. Let $z_{1}$ and $z_{2}$ be points of $X$ such that both $\mathcal{T}\left(\left\{z_{1}\right\}\right)$ and $\mathcal{T}\left(\left\{z_{2}\right\}\right)$ have property $B L$. Suppose $\mathcal{T}\left(\left\{z_{1}\right\}\right) \cap \mathcal{T}\left(\left\{z_{2}\right\}\right) \neq \emptyset$. Let $z_{3} \in \mathcal{T}\left(\left\{z_{1}\right\}\right) \cap \mathcal{T}\left(\left\{z_{2}\right\}\right)$. Then, by Lemma 3.6, $\mathcal{T}\left(\left\{z_{1}\right\}\right)=\mathcal{T}\left(\left\{z_{3}\right\}\right)=\mathcal{T}\left(\left\{z_{2}\right\}\right)$

Lemma 3.8. Let $X$ be a decomposable continuum for which $\mathcal{T}$ is idempotent on singletons. If $z \in X$, then

$$
\mathcal{T}(\{z\})=\bigcup\{\mathcal{T}(\{w\}) \mid w \in \mathcal{T}(\{z\})\}
$$

Proof. Let $z \in X$ and let $w_{0} \in \mathcal{T}(\{z\})$. Since $\mathcal{T}$ is idempotent on singletons $\mathcal{T}\left(\left\{w_{0}\right\}\right) \subset$ $\mathcal{T}(\mathcal{T}(\{z\}))=\mathcal{T}(\{z\})$. Hence, $\bigcup\{\mathcal{T}(\{w\}) \mid w \in \mathcal{T}(\{z\})\} \subset \mathcal{T}(\{z\})$. The other inclusion is clear.

The proof of the following theorem is based on a technique of Bellamy and Lum [1, Lemma 5].

Theorem 3.9. If $X$ is a decomposable continuum for which $\mathcal{T}$ is idempotent on singletons, then for each $x \in X$, there exists $z \in \mathcal{T}(\{x\})$ such that $\mathcal{T}(\{z\})$ has property $B L$.

Proof. Let $x \in X$. By Lemma 3.8, we have that

$$
\mathcal{T}(\{x\})=\bigcup\{\mathcal{T}(\{w\}) \mid w \in \mathcal{T}(\{z\})\} .
$$

Let $\mathcal{G}_{x}=\{\mathcal{T}(\{w\}) \mid w \in \mathcal{T}(\{x\})\} . \quad$ Partially order $\mathcal{G}_{x}$ by inclusion. Let $\mathcal{H}=$ $\left\{\mathcal{T}\left(\left\{w_{\lambda}\right\}\right)\right\}_{\lambda \in \Lambda}$ be a (set theoretic) chain of elements of $\mathcal{G}_{x}$. We show that $\mathcal{H}$ has a lower bound in $\mathcal{G}_{x}$.

Since $\mathcal{H}$ is a chain of continua (Theorem 3.5), $\bigcap_{\lambda \in \Lambda} \mathcal{T}\left(\left\{w_{\lambda}\right\}\right) \neq \emptyset$. Let $w_{0} \in$ $\bigcap_{\lambda \in \Lambda} \mathcal{T}\left(\left\{w_{\lambda}\right\}\right)$. Then

$$
\begin{aligned}
\mathcal{T}\left(\left\{w_{0}\right\}\right) \subset \mathcal{T} & \left(\bigcap_{\lambda \in \Lambda} \mathcal{T}\left(\left\{w_{\lambda}\right\}\right)\right) \subset \bigcap_{\lambda \in \Lambda} \mathcal{T}\left(\mathcal{T}\left(\left\{w_{\lambda}\right\}\right)\right)= \\
& \bigcap_{\lambda \in \Lambda} \mathcal{T}\left(\left\{w_{\lambda}\right\}\right) \subset \mathcal{T}(\{x\}) .
\end{aligned}
$$

Hence, by Kuratowski-Zorn Lemma, there exists $z \in \mathcal{T}(\{x\})$ such that $\mathcal{T}(\{z\})$ is a minimal element; i.e., each $w \in \mathcal{T}(\{z\})$ satisfies that $\mathcal{T}(\{z\}) \subset \mathcal{T}(\{w\})$. Therefore, $\mathcal{T}(\{z\})$ has property $B L$.

Lemma 3.10. Let $X$ and $Y$ be continua and let $f: X \rightarrow Y$ be a surjective map. If $f$ is monotone, then $\mathcal{T}_{Y}(B)=f \mathcal{T}_{X} f^{-1}(B)$, for all $B \in 2^{Y}$.

Vol. 37, $\left.\mathrm{N}^{\circ} 1,2019\right]$ 
Proof. Let $y \in Y \backslash f \mathcal{T}_{X} f^{-1}(B)$. Then $f^{-1}(y) \cap \mathcal{T}_{X} f^{-1}(B)=\emptyset$. Thus, for each $x \in f^{-1}(y)$, there exists a subcontinuum $W_{x}$ of $X$ such that $x \in \operatorname{Int}\left(W_{x}\right) \subset W_{x} \subset X \backslash f^{-1}(B)$. Since $f^{-1}(y)$ is compact, there exist $x_{1}, \ldots, x_{n} \in f^{-1}(y)$ such that $f^{-1}(y) \subset \bigcup_{j=1}^{n} \operatorname{Int}\left(W_{x_{j}}\right)$. Note that $\left(\bigcup_{j=1}^{n} W_{x_{j}}\right) \cap f^{-1}(B)=\emptyset$, and for each $j \in\{1, \ldots, n\}, f^{-1}(y) \cap W_{x_{j}} \neq \emptyset$. Hence, $f\left(\bigcup_{j=1}^{n} W_{x_{j}}\right) \cap B=\emptyset$ and $f\left(\bigcup_{j=1}^{n} W_{x_{j}}\right)$ is a continuum $\left(y \in f\left(W_{x_{j}}\right) \cap f\left(W_{x_{k}}\right)\right.$ for every $j, k \in\{1,2, \ldots, n\})$.

Observe that $Y \backslash f\left(X \backslash \bigcup_{j=1}^{n} \operatorname{Int}\left(W_{x_{j}}\right)\right)$ is an open set of $Y$ and it is contained in $f\left(\bigcup_{j=1}^{n} W_{x_{j}}\right)$. To see this, note that, since $\bigcup_{j=1}^{n} \operatorname{Int}\left(W_{x_{j}}\right) \subset \bigcup_{j=1}^{n} W_{x_{j}}, X \backslash \bigcup_{j=1}^{n} W_{x_{j}} \subset$ $X \backslash \bigcup_{j=1}^{n} \operatorname{Int}\left(W_{x_{j}}\right)$. Then $f\left(X \backslash \bigcup_{j=1}^{n} W_{x_{j}}\right) \subset f\left(X \backslash \bigcup_{j=1}^{n} \operatorname{Int}\left(W_{x_{j}}\right)\right)$ and, consequently, $Y \backslash f\left(X \backslash \bigcup_{j=1}^{n} \operatorname{Int}\left(W_{x_{j}}\right)\right) \subset Y \backslash f\left(X \backslash \bigcup_{j=1}^{n} W_{x_{j}}\right)$. Since $f$ is a surjection, $Y \backslash f\left(X \backslash \bigcup_{j=1}^{n} \operatorname{Int}\left(W_{x_{j}}\right)\right) \subset f\left(X \backslash\left(X \backslash \bigcup_{j=1}^{n} W_{x_{j}}\right)\right)=f\left(\bigcup_{j=1}^{n} W_{x_{j}}\right)$. Thus,

$$
Y \backslash f\left(X \backslash \bigcup_{j=1}^{n} \operatorname{Int}\left(W_{x_{j}}\right)\right) \subset f\left(\bigcup_{j=1}^{n} W_{x_{j}}\right) .
$$

Also, since $f^{-1}(y) \subset \bigcup_{j=1}^{n} \operatorname{Int}\left(W_{x_{j}}\right)$, we have $\{y\} \cap f\left(X \backslash \bigcup_{j=1}^{n} \operatorname{Int}\left(W_{x_{j}}\right)\right)=\emptyset$. Hence, $y \in Y \backslash f\left(X \backslash \bigcup_{j=1}^{n} \operatorname{Int}\left(W_{x_{j}}\right)\right)$. Therefore, $y \in Y \backslash \mathcal{T}_{Y}(B)$.

Let $x \in X \backslash f^{-1} \mathcal{T}_{Y}(B)$. Then $f(x) \in Y \backslash \mathcal{T}_{Y}(B)$. Hence, there exists a subcontinuum $W$ of $Y$ such that $f(x) \in \operatorname{Int}(W) \subset W \subset Y \backslash B$. This implies that $x \in f^{-1}(f(x)) \subset$ $f^{-1}(\operatorname{Int}(W)) \subset f^{-1}(W) \subset X \backslash f^{-1}(B)$. Since $f$ is monotone, $f^{-1}(W)$ is a subcontinuum of $X$. Therefore, $x \in X \backslash \mathcal{T}_{X} f^{-1}(B)$.

We know that $\mathcal{T}_{Y}(B) \subset f \mathcal{T}_{X} f^{-1}(B)$. Since $\mathcal{T}_{X} f^{-1}(B) \subset f^{-1} \mathcal{T}_{Y}(B)$, by previouse paragraph, $f \mathcal{T}_{X} f^{-1}(B) \subset f f^{-1} \mathcal{T}_{Y}(B)=\mathcal{T}_{Y}(B)$. Therefore, $f \mathcal{T}_{X} f^{-1}(B)=\mathcal{T}_{Y}(B)$.

\section{Property of Kelley}

The property of Kelley for Hausdorff continua is defined independently in [3] and [14]. In [3], a homogeneous continuum without the property of Kelley is given, showing that the fact that homogeneous metric continua have the property of Kelley $([8,4.2 .36]$ or $[19,(2.5)])$ is not true in this more general setting. It is also mentioned that hereditarily indecomposable continua have the property of Kelley [3, Proposition 2.7]. In [14], the property of Kelley is used to study points of various types of local connectedness in the hyperspace of subcontinua of a continuum. In fact, if a continuum $X$ has the property of Kelley, then local connectedness of $\mathcal{C}_{1}(X)$ is proved to be equivalent to some stronger kinds of local connectedness and of local arcwise connectedness [14].

A continuum $X$ has the property of Kelley at a point $x_{0}$ if for every subcontinuum $K$ of $X$ containing $x_{0}$ and each open subset $\mathcal{U}$ of $\mathcal{C}_{1}(X)$ containing $K$, there exists an open subset $V$ of $X$ such that $x_{0} \in V$, and if $x \in V$, then there exists a subcontinuum $L$ of $X$ such that $x \in L$ and $L \in \mathcal{U}$. The open set $V$ is called a Kelley set for $\mathcal{U}$ and $x_{0}$. The 
continuum $X$ has the property of Kelley if $X$ has the property of Kelley at each of its points.

Theorem 4.1. Let $X$ be a continuum with the property of Kelley. Let $A$ be a nonempty closed subset of $X$ and let $W$ be a subcontinuum of $X$ such that $\operatorname{Int}(W) \neq \emptyset$ and $W \cap A=$ $\emptyset$. If $w \in W$, then there exists a subcontinuum $K$ of $X$ such that $w \in \operatorname{Int}(K)$ and $K \cap A=\emptyset$.

Proof. Let $w \in W$. Since $X$ is normal, there exists an open subset $U$ of $X$ such that $W \subset U$ and $C l(U) \cap A=\emptyset$. Let $\mathcal{U}=\langle U, \operatorname{Int}(W)\rangle_{1}$. Note that $W \in \mathcal{U}$. Also observe that if $L \in \mathcal{U}$, then $L \subset U$ and $L \cap W \neq \emptyset$. Since $X$ has the property of Kelley at $w$, there exists a Kelley set $V$ for $\mathcal{U}$ and $w$. Let $v \in V \backslash\{w\}$. Then there exists a subcontinuum $L_{v}$ of $X$ such that $v \in L_{v}$ and $L_{v} \in \mathcal{U}$. Let $L_{w}=W$, and let $K=C l\left(\bigcup\left\{L_{v} \mid v \in V\right\}\right)$. Then $K$ is a subcontinuum of $X, w \in V \subset K$ and $K \subset C l(U) \subset X \backslash A$.

Corollary 4.2. Let $X$ be a continuum with the property of Kelley, let $W$ be a subcontinuum of $X$, with non-empty interior, and let $A$ be a non-empty closed subset of $X$ such that $W \cap A=\emptyset$. Then there exists a subcontinuum $M$ of $X$ such that $W \subset \operatorname{Int}(M)$ and $M \cap A=\emptyset$.

Proof. Let $w \in W$. By Theorem 4.1, there exists a subcontinuum $K_{w}$ such that $w \in$ $\operatorname{Int}(K)$ and $K \cap A=\emptyset$. Note that $\left\{\operatorname{Int}\left(K_{w}\right) \mid w \in W\right\}$ forms an open cover of $W$, so that by compactness, there exist $w_{1}, \ldots, w_{n}$ in $W$ such that $W \subset \bigcup_{j=1}^{n} \operatorname{Int}\left(K_{w_{j}}\right)$. Let $M=\bigcup_{j=1}^{n} K_{w_{j}}$. Then $M$ is a subcontinuum of $X, W \subset \operatorname{Int}(M)$ and $M \cap A=\emptyset$.

The proof of the following result is different from the one given in [12, Theorem 5.2].

Theorem 4.3. Let $X$ be a continuum with the property of Kelley and let $W_{1}$ and $W_{2}$ be disjoint subcontinua with non-empty interior. Then $\mathcal{T}\left(W_{1}\right) \cap \mathcal{T}\left(W_{2}\right)=\emptyset$.

Proof. By Corollary 4.2, there exists a subcontinuum $M_{1}$ such that $W_{1} \subset \operatorname{Int}\left(M_{1}\right)$ and $M_{1} \cap W_{2}=\emptyset$. This implies that $W_{1} \cap \mathcal{T}\left(W_{2}\right)=\emptyset$. By Corollary 4.2, there exists a subcontinuum $M_{2}$ such that $\mathcal{T}\left(W_{2}\right) \subset \operatorname{Int}\left(M_{2}\right)$ and $M_{2} \cap W_{1}=\emptyset$. Hence, $\mathcal{T}\left(W_{2}\right) \cap$ $\mathcal{T}\left(W_{1}\right)=\emptyset$.

Theorem 4.4. If $X$ is a continuum with the property of Kelley, then $\mathcal{T}$ is idempotent on closed sets.

Proof. Let $A$ be a nonempty closed set of $X$. We know that $\mathcal{T}(A) \subset \mathcal{T}^{2}(A)$. Let $x \in X \backslash \mathcal{T}(A)$. Then there exists a subcontinuum $W$ of $X$ such that $x \in \operatorname{Int}(W) \subset W \subset$ $X \backslash A$. By Corollary 4.2, there exists a subcontinuum $M$ of $X$ such that $W \subset \operatorname{Int}(M)$ and $M \cap A=\emptyset$. Since $\operatorname{Int}(M) \subset X \backslash \mathcal{T}(A), x \in \operatorname{Int}(W) \subset W \subset X \backslash \mathcal{T}(A)$. Hence, $x \in X \backslash \mathcal{T}^{2}(A)$. Therefore, $\mathcal{T}$ is idempotent on closed sets.

Remark 4.5. Theorem 4.4 cannot be improved to obtain that the function $\mathcal{T}$ is idempotent, even for metric continua. Let $X$ be the product of a solenoid and a simple closed curve. Then $X$ is a homogeneous metric continuum. By [8, 4.2.32], $\mathcal{T}_{X}$ is idempotent on closed sets and, by [10, Theorem 3.3], $\mathcal{T}_{X}$ is not idempotent.

Vol. 37, $\left.\mathrm{N}^{\circ} 1,2019\right]$ 
Theorem 4.6. Let $X$ be a continuum. If $\mathcal{G}=\left\{\mathcal{T}_{X}(\{x\}) \mid x \in X\right\}$ is a decomposition of $X$, then $\mathcal{G}$ is upper semicontinuous. Hence, $X / \mathcal{G}$ is a continuum.

Proof. By Lemma 3.3, $\mathcal{T}$ is upper semicontinuous. Hence, $\mathcal{G}$ is an upper semicontinuous decomposition. To show that $X / \mathcal{G}$ is Hausdorff, let $q: X \rightarrow X / \mathcal{G}$ be the quotient map and let $\chi_{1}$ and $\chi_{2}$ be two distinct points of $X / \mathcal{G}$. Then $q^{-1}\left(\chi_{1}\right)$ and $q^{-1}\left(\chi_{2}\right)$ are two disjoint closed subsets of $X$. Since $X$ is normal, there exist two disjoint open subsets $U_{1}$ and $U_{2}$ of $X$ such that $q^{-1}\left(\chi_{1}\right) \subset U_{1}$ and $q^{-1}\left(\chi_{2}\right) \subset U_{2}$. Note that, since $\mathcal{G}$ is an upper semicontinuous decomposition, there exist two saturated open subsets $W_{U_{1}}$ and $W_{U_{2}}$ of $X$ such that $q^{-1}\left(\chi_{1}\right) \subset W_{U_{1}} \subset U_{1}$ and $q^{-1}\left(\chi_{2}\right) \subset W_{U_{2}} \subset U_{2}$. Then $q\left(W_{U_{1}}\right)$ and $q\left(W_{U_{2}}\right)$ are two disjoint open subsets of $X / \mathcal{G}$ such that $\chi_{1} \in q\left(W_{U_{1}}\right)$ and $\chi_{2} \in q\left(W_{U_{2}}\right)$. Therefore, $X / \mathcal{G}$ is a Hausdorff space.

Corollary 4.7. Let $X$ be a continuum such that $\mathcal{T}_{X}$ is idempotent on singletons. If $\mathcal{G}=$ $\left\{\mathcal{T}_{X}(\{x\}) \mid x \in X\right\}$ is a decomposition of $X$, then $X / \mathcal{G}$ is an aposyndetic continuum.

Proof. By Theorem 4.6, $\mathcal{G}$ is an upper semicontinuous decomposition and $X / \mathcal{G}$ is a continuum. Let $q: X \rightarrow X / \mathcal{G}$ be the quotient map. Then $q$ is a monotone map. Let $\chi \in X / \mathcal{G}$ and let $x \in X$ be such that $\mathcal{T}_{X}(\{x\})=q^{-1}(\chi)$. Since $q$ is a monotone map, by Lemma 3.10, $\mathcal{T}_{X / \mathcal{G}}(\{\chi\})=q \mathcal{T}_{X} q^{-1}(\chi)=q \mathcal{T}_{X}\left(\mathcal{T}_{X}(\{x\})\right)=q \mathcal{T}_{X}(\{x\})=q q^{-1}(\chi)=\{\chi\} ;$ the third equality is true because $\mathcal{T}_{X}$ is idempotent on singletons. Therefore, $X / \mathcal{G}$ is an aposyndetic continuum.

Theorem 4.8. Let $X$ be a continuum with the property of Kelley. If $\mathcal{G}=\left\{\mathcal{T}_{X}(\{x\}) \mid x \in\right.$ $X\}$ is a decomposition of $X$, then $\mathcal{T}(\{x\})$ is a terminal subcontinuum of $X$ for all $x \in X$.

Proof. Suppose there exists a point $x_{0}$ in $X$ such that $\mathcal{T}\left(\left\{x_{0}\right\}\right)$ is not a terminal subcontinuum of $X$. Then there exists a subcontinuum $L$ of $X$ such that $L \cap \mathcal{T}\left(\left\{x_{0}\right\}\right) \neq \emptyset$, $L \backslash \mathcal{T}\left(\left\{x_{0}\right\}\right) \neq \emptyset$ and $\mathcal{T}\left(\left\{x_{0}\right\}\right) \backslash L \neq \emptyset$. Let $p \in L \backslash \mathcal{T}\left(\left\{x_{0}\right\}\right)$ and $\ell \in L \cap \mathcal{T}\left(\left\{x_{0}\right\}\right)$. Since $\mathcal{G}$ is a decomposition, without loss of generality, we assume that $x_{0} \in \mathcal{T}\left(\left\{x_{0}\right\}\right) \backslash L$. Since $p \in L \backslash \mathcal{T}\left(\left\{x_{0}\right\}\right)$, there exists a subcontinuum $W$ of $X$ such that $p \in \operatorname{Int}(W) \subset$ $W \subset X \backslash\left\{x_{0}\right\}$. Let $K=L \cup W$. Then $K$ is a subcontinuum of $X, p \in \operatorname{Int}(K)$ and $K \subset X \backslash\left\{x_{0}\right\}$. By Theorem 4.1, there exists a subcontinuum $M$ of $X$ such that $\ell \in \operatorname{Int}(M)$ and $M \subset X \backslash\left\{x_{0}\right\}$, a contradiction to the choice of $\ell$. Therefore, $\mathcal{T}(\{x\})$ is a terminal subcontinuum of $X$ for all $x \in X$.

Let $X$ and $Z$ be continua and let $f: X \rightarrow Z$ be a map. Then, $f$ is an atomic map if for each subcontinuum $K$ of $X$ such that $f(K)$ is not degenerate, then $K=f^{-1}(f(K))$.

Next we prove a weak version of F. Burton Jones Aposyndetic Decomposition Theorem. Note that we obtain that the decomposition is only upper semicontinuous.

Theorem 4.9. Let $X$ be a homogeneous continuum with the property of Kelley. If $\mathcal{G}=$ $\{\mathcal{T}(\{x\}) \mid x \in X\}$, then $\mathcal{G}$ is an upper semicontinuous terminal decomposition of $X$, $X / \mathcal{G}$ is an aposyndetic homogeneous continuum, and the quotient map $q: X \rightarrow X / \mathcal{G}$ is an atomic map.

[Revista Integración, temas de matemáticas 
Proof. Let $X$ be a point of $X$. We show that if $z \in \mathcal{T}(\{x\})$, then $\mathcal{T}(\{z\})=\mathcal{T}(\{x\})$. Since $X$ the property of Kelley, by Theorem 4.4, $\mathcal{T}$ is idempotent on closed sets. Hence, $\mathcal{T}(\{z\}) \subset \mathcal{T}(\{x\})$. Also, by Theorem 3.9, there exists $x_{0} \in \mathcal{T}(\{x\})$ such that $\mathcal{T}\left(\left\{x_{0}\right\}\right)$ has property $B L$. Since $X$ is homogeneous, there exists a homeomorphism $h: X \rightarrow X$ such that $h\left(x_{0}\right)=x$. Since $z \in \mathcal{T}(\{x\}), h^{-1}(z) \in \mathcal{T}\left(\left\{x_{0}\right\}\right)$. Hence, by Lemma 3.6, $\mathcal{T}\left(\left\{h^{-1}(z)\right\}\right)=\mathcal{T}\left(\left\{x_{0}\right\}\right)$. Thus, by Lemma 3.4, $\mathcal{T}(\{x\})=h\left(\mathcal{T}\left(\left\{x_{0}\right\}\right)\right)=$ $h\left(\mathcal{T}\left(\left\{h^{-1}(z)\right\}\right)\right)=\mathcal{T}(\{z\})$. Therefore, $\mathcal{G}$ is a decomposition of $X$. By Theorem 4.6, $\mathcal{G}$ is upper semicontinuous. The fact that each element of $\mathcal{G}$ is a terminal subcontinuum of $X$ follows from Theorem 4.8. Also, by Corollary $4.7, X / \mathcal{G}$ is an aposyndetic continuum. We show that $X / \mathcal{G}$ is homogeneous. Let $q: X \rightarrow X / \mathcal{G}$ be the quotient map. Let $\chi_{1}$ and $\chi_{2}$ be two elements of $X / \mathcal{G}$. We define $\zeta: X / \mathcal{G} \rightarrow X / \mathcal{G}$ as follows: Let $x_{1}$ and $x_{2}$ be elements of $X$ such that $q\left(x_{1}\right)=\chi_{1}$ and $q\left(x_{2}\right)=\chi_{2}$. Since $X$ is homogeneous, there exists a homeomorphism $h: X \rightarrow X$ such that $h\left(x_{1}\right)=x_{2}$. Note that, by Lemma 3.4, $h\left(\mathcal{T}\left(\left\{x_{1}\right\}\right)=\mathcal{T}\left(\left\{x_{2}\right\}\right)\right.$; i.e., $h\left(q^{-1}\left(\chi_{1}\right)\right)=q^{-1}\left(\chi_{2}\right)$. Define

$$
\zeta(\chi)=q \circ h\left(q^{-1}(\chi)\right) .
$$

Then $\zeta$ is well defined and $\zeta\left(\chi_{1}\right)=\chi_{2}$. To see that $\zeta$ is continuous, let $\mathcal{U}$ be an open subset $X / \mathcal{G}$. Then $q^{-1}(\mathcal{U})$ is a saturated open subset of $X$. Since $h$ is a homeomorphism, $h^{-1}\left(q^{-1}(\mathcal{U})\right)$ is a saturated open subset of $X$. Hence, $q\left(h^{-1}\left(q^{-1}(\mathcal{U})\right)\right)=\zeta^{-1}(\mathcal{U})$ is an open subset of $X / \mathcal{G}$. Thus, $\zeta$ is continuous. Similarly, $\zeta^{-1}$ is defined and is continuous. Therefore, $X / \mathcal{G}$ is homogeneous. To see the quotient map is atomic, let $K$ be a subcontinuum of $X$ such that $q(K)$ is nondegenerate. Clearly, $K \subset q^{-1} q(K)$. Let $x \in q^{-1} q(K)$. Then there exists $k \in K$ such that $q(k)=q(x)$. Thus, $q^{-1} q(x) \cap K \neq \emptyset$. Since $q^{-1} q(x)$ is a terminal subcontinuum of $X$, from Theorem 4.8 and the fact that $q(K)$ is nondegenerate, we have that $x \in q^{-1} q(x) \subset K$. Hence, $q^{-1} q(K) \subset K$. Therefore, $q$ is an atomic map.

In the following theorem we extend to (nonmetric) continua [2, Theorem 5.4].

Theorem 4.10. Let $X$ be a decomposable continuum with the property of Kelley. If $\mathcal{T}_{X}$ is continuous on singletons and

$$
\mathcal{G}=\left\{\mathcal{T}_{X}(\{x\}) \mid x \in X\right\},
$$

then $\mathcal{T}_{X}(Z)=q^{-1} \mathcal{T}_{X / \mathcal{G}} q(Z)$ for each nonempty closed subset $Z$ of $X$, where $q: X \rightarrow X / \mathcal{G}$ is the quotient map.

Proof. Without loss of generality, we assume that $X$ is not aposyndetic. Note that by Theorem 4.4 and [11, Theorem 3.4], $\mathcal{G}$ is a decomposition of $X$. Let $Z$ be a nonempty closed subset of $X$. We divide the proof in six steps.

Step 1. $q^{-1} q(Z) \subset \mathcal{T}_{X}(Z)$.

Let $x \in q^{-1} q(Z)$. Then $q(x) \in q(Z)$. Thus, there exists $z \in Z$ such that $q(z)=q(x)$. This implies that $\mathcal{T}_{X}(\{z\})=\mathcal{T}_{X}(\{x\})$. Hence, since $\mathcal{T}_{X}$ is idempotent on closed sets (Theorem 4.4), $\mathcal{T}_{X}(\{x\})=\mathcal{T}_{X}(\{z\}) \subset \mathcal{T}_{X}(Z)$. Therefore, $x \in \mathcal{T}_{X}(Z)$, and $q^{-1} q(Z) \subset$ $\mathcal{T}_{X}(Z)$.

Vol. 37, $\left.\mathrm{N}^{\circ} 1,2019\right]$ 
Step 2. $\mathcal{T}_{X}(Z)=q^{-1} q \mathcal{T}_{X}(Z)$.

Clearly, $\mathcal{T}_{X}(Z) \subset q^{-1} q \mathcal{T}_{X}(Z)$. Let $x \in q^{-1} q \mathcal{T}_{X}(Z)$. Then $q(x) \in q \mathcal{T}_{X}(Z)$. Hence, there exists $y \in \mathcal{T}_{X}(Z)$ such that $q(y)=q(x)$. This implies that $\mathcal{T}_{X}(\{y\})=\mathcal{T}_{X}(\{x\})$. Thus, since $\mathcal{T}_{X}$ is idempotent on closed sets (Theorem 4.4), we have that $\mathcal{T}_{X}(\{y\}) \subset \mathcal{T}_{X}(Z)$. Hence, $x \in \mathcal{T}_{X}(Z)$, and $q^{-1} q \mathcal{T}_{X}(Z) \subset \mathcal{T}_{X}(Z)$. Therefore, $\mathcal{T}_{X}(Z)=q^{-1} q \mathcal{T}_{X}(Z)$.

Step 3. If $Z$ is connected and $\operatorname{Int}_{X}(Z) \neq \emptyset$, then $Z=q^{-1} q(Z)$.

Clearly, $Z \subset q^{-1} q(Z)$. Let $x \in q^{-1} q(Z)$. Then $q(x) \in q(Z)$. Thus, there exists $z \in Z$ such that $q(z)=q(x)$. This implies that $\mathcal{T}_{X}(\{z\})=\mathcal{T}_{X}(\{x\})$. Since $\mathcal{T}_{X}(\{x\})$ is a nowhere dense terminal subcontinuum of $X$ (Lemma 3.2, Lemma 3.4 and Theorem 3.5), $\mathcal{T}_{X}(\{x\}) \cap Z \neq \emptyset$ and $\operatorname{Int}_{X}(Z) \neq \emptyset$, we have that $\mathcal{T}_{X}(\{x\}) \subset Z$. In particular, $x \in Z$. Therefore, $Z=q^{-1} q(Z)$.

Step 4. $q \mathcal{T}_{X}(Z) \subset \mathcal{T}_{X / \mathcal{G}} q(Z)$.

Let $\chi \in X / \mathcal{G} \backslash \mathcal{T}_{X / \mathcal{G}} q(Z)$. Then there exists a subcontinuum $\mathcal{W}$ of $X / \mathcal{G}$ such that $\chi \in \operatorname{Int}_{X / \mathcal{G}}(\mathcal{W}) \subset \mathcal{W} \subset X / \mathcal{G} \backslash q(Z)$. From these inclusions we obtain that $q^{-1}(\chi) \subset$ $\operatorname{Int}_{X}\left(q^{-1}(\mathcal{W})\right) \subset q^{-1}(\mathcal{W}) \subset X \backslash q^{-1} q(Z) \subset X \backslash Z$. Hence, since $q$ is monotone (Theorem 4.9), $q^{-1}(\chi) \cap \mathcal{T}_{X}(Z)=\emptyset$. Thus, $q q^{-1}(\chi) \cap q \mathcal{T}_{X}(Z)=\emptyset$. Therefore, $\chi \in X / \mathcal{G} \backslash q \mathcal{T}_{X}(Z)$, and $q \mathcal{T}_{X}(Z) \subset \mathcal{T}_{X / \mathcal{G}} q(Z)$.

Step 5. $\mathcal{T}_{X / \mathcal{G}} q(Z) \subset q \mathcal{T}_{X}(Z)$.

Let $\chi \in X / \mathcal{G} \backslash q \mathcal{T}_{X}(Z)$. Then $\{\chi\} \cap q \mathcal{T}_{X}(Z)=\emptyset$. This implies that $q^{-1}(\chi) \cap q^{-1} q \mathcal{T}_{X}(Z)=$ $\emptyset$. Hence, by Step $2, q^{-1}(\chi) \cap \mathcal{T}_{X}(Z)=\emptyset$. Since $\mathcal{T}_{X}$ is idempotent on closed sets (Theorem 4.4), $q^{-1}(\chi) \cap \mathcal{T}_{X}^{2}(Z)=\emptyset$. Thus, there exists a subcontinuum $W$ of $X$ such that $q^{-1}(\chi) \subset \operatorname{Int}_{X}(W) \subset W \subset X \backslash \mathcal{T}_{X}(Z) \subset X \backslash Z$. From these inclusions, since $q$ is an open map ( $\mathcal{G}$ is a continuous decomposition), we obtain that $\{\chi\}=q q^{-1}(\chi) \subset$ $\operatorname{Int}_{X / \mathcal{G}}(q(W)) \subset q(W) \subset q(X \backslash Z)$. To finish, we need to show that $q(W) \cap q(Z)=\emptyset$. Suppose there exists $\chi^{\prime} \in q(W) \cap q(Z)$. Then, by Steps 3 and $1, q^{-1}\left(\chi^{\prime}\right) \subset q^{-1} q(W) \cap$ $q^{-1} q(Z)=W \cap q^{-1} q(Z) \subset W \cap \mathcal{T}_{X}(Z)$, a contradiction to the election of $W$. Hence, $q(W) \cap q(Z)=\emptyset$, and $\chi \in X / \mathcal{G} \backslash \mathcal{T}_{X / \mathcal{G}} q(Z)$. Therefore, $\mathcal{T}_{X / \mathcal{G}} q(Z) \subset q \mathcal{T}_{X}(Z)$.

Step 6. $q \mathcal{T}_{X}(Z)=\mathcal{T}_{X / \mathcal{G}} q(Z)$.

The equality follows from Steps 4 and 5 .

From Steps 2 and 6, we have that

$$
\mathcal{T}_{X}(Z)=q^{-1} q \mathcal{T}_{X}(Z)=q^{-1} \mathcal{T}_{X / \mathcal{G}} q(Z)
$$

Therefore, $\mathcal{T}_{X}(Z)=q^{-1} \mathcal{T}_{X / \mathcal{G}} q(Z)$.

The property of Kelley weakly is introduced in [13] to study points of connectedness im kleinen of the $n$-fold hyperspaces of metric continua. Next, we extend this property to continua.

[Revista Integración, temas de matemáticas 
A continuum $X$ has the property of Kelley weakly, if there exists a dense subset $\mathcal{A}$ of $\mathcal{C}_{1}(X)$ such that $X$ has the property of Kelley at some point of each element $A \in \mathcal{A}$.

Let $X$ be a continuum and let $n$ be a positive integer. Then $\mathcal{C}_{n}(X)$ denotes the $n$-fold hyperspace of $X$; i.e.,

$$
\mathcal{C}_{n}(X)=\left\{A \in 2^{X} \mid A \text { has at most } n \text { components }\right\},
$$

with the Vietoris topology. If $U_{1}, \ldots, U_{m}$ are open subsets of $X$, then $\left\langle U_{1}, \ldots, U_{m}\right\rangle \cap$ $\mathcal{C}_{n}(X)$ is denoted by $\left\langle U_{1}, \ldots, U_{m}\right\rangle_{n}$.

Lemma 4.11. Let $X$ be a continuum with the property of Kelley weakly and let $n$ be a positive integer. Then for each open subset $\mathcal{U}$ of $\mathcal{C}_{n}(X)$, there exists $A \in \mathcal{U}$ such that $X$ has the property of Kelley at some point a of A.

Proof. Let $\mathcal{U}$ be an open subset of $\mathcal{C}_{n}(X)$, let $K \in \mathcal{U}$ and let $V_{1}, \ldots, V_{t}$ be open subsets of $X$ such that $K \in\left\langle V_{1}, \ldots, V_{t}\right\rangle_{n} \subset \mathcal{U}$. Let $L$ be a component of $K$ and let $V_{j 1}, \ldots, V_{j l}$ denote all the elements of $\left\{V_{1}, \ldots, V_{t}\right\}$ that $L$ intersects. Then $L \in\left\langle V_{j 1}, \ldots, V_{j l}\right\rangle_{1}$. Since $X$ has the property of Kelley weakly, there exists $M \in \mathcal{C}_{1}(X)$ such that $M \in$ $\left\langle V_{j 1}, \ldots, V_{j l}\right\rangle_{1}$ and $X$ has the property of Kelley at some point $a$ of $M$. Let $A=(K \backslash$ $L) \cup M$. Then $A \in\left\langle V_{1}, \ldots, V_{t}\right\rangle_{n} \subset \mathcal{U}$ and $X$ has the property of Kelley at $a \in M \subset A$. $\square$

Part (2) of the following theorem is known for the hyperspace of subcontinua of a continuum [14, Theorem 11].

Theorem 4.12. Let $X$ be a continuum and let $\mathcal{U}$ be an open subset of $\mathcal{C}_{n}(X)$.

(1) If $X$ has the property of Kelley weakly, then Int $(\bigcup \mathcal{U}) \neq \emptyset$.

(2) If $X$ has the property of Kelley, then $\bigcup \mathcal{U}$ is an open subset of $X$.

Proof. We prove (1). By Lemma 4.11, there exists $A \in \mathcal{U}$ such that $X$ has the property of Kelley at some point $a$ of $A$. Let $V_{1}, \ldots, V_{t}$ be open subsets of $X$ such that $A \in$ $\left\langle V_{1}, \ldots, V_{t}\right\rangle_{n} \subset \mathcal{U}$. Let $A_{1}$ be the component of $A$ containing $a$. Let $V_{j 1}, \ldots, V_{j l}$ denote all the elements of $\left\{V_{1}, \ldots, V_{t}\right\}$ that $A_{1}$ intersects. Since $X$ has the property of Kelley at $a$, there exists a Kelley set $V$ for $a$ and $\left\langle V_{j 1}, \ldots, V_{j l}\right\rangle_{1}$. We show that $V \subset \cup \mathcal{U}$. Let $z \in V$. Then there exists $D \in \mathcal{C}_{1}(X)$ such that $z \in D$ and $D \in\left\langle V_{j 1}, \ldots, V_{j l}\right\rangle_{1}$. Let $B=\left(A \backslash A_{1}\right) \cup D$. Then $B \in \mathcal{C}_{n}(X)$ and $B \in\left\langle V_{1}, \ldots, V_{t}\right\rangle_{n} \subset \mathcal{U}$. Hence, $B \subset \cup \mathcal{U}$. Since $z \in D \subset B, z \in \bigcup \mathcal{U}$, and $V \subset \bigcup \mathcal{U}$. Therefore, $\operatorname{Int}(\bigcup \mathcal{U}) \neq \emptyset$.

To prove (2), let $x \in \cup \mathcal{U}$. Then there exists $C \in \mathcal{U}$ such that $x \in C$. Let $C_{1}, \ldots, C_{t}$ be the components of $C$. Without loss of generality, we assume that $x \in C_{1}$. Let $V_{1}, \ldots, V_{t}$ be open subsets of $X$ such that $C \in\left\langle V_{1}, \ldots, V_{t}\right\rangle_{n} \subset \mathcal{U}$. Let $V_{j 1}, \ldots, V_{j l}$ denote all the elements of $\left\{V_{1}, \ldots, V_{t}\right\}$ that $C_{1}$ intersects. Since $X$ has the property of Kelley at $x$, there exists a Kelley set $V$ for $x$ and $\left\langle V_{j 1}, \ldots, V_{j l}\right\rangle_{1}$. We show that $V \subset \cup \mathcal{U}$. Let $z \in V$. Then there exists $L \in \mathcal{C}_{1}(X)$ such that $z \in L$ and $L \in\left\langle V_{j 1}, \ldots, V_{j l}\right\rangle_{1}$. Let $R=\left(C \backslash C_{1}\right) \cup L$. Then $R \in \mathcal{C}_{n}(X)$ and $R \in\left\langle V_{1}, \ldots, V_{t}\right\rangle_{n} \subset \mathcal{U}$. Since $z \in L \subset R, z \in \bigcup \mathcal{U}$ and $V \subset \bigcup \mathcal{U}$. Thus, $x$ is an interior point of $\bigcup \mathcal{U}$. Therefore, $\bigcup \mathcal{U}$ is open in $X$.

Lemma 4.13. Let $X$ be a continuum. If $\mathcal{K}$ is a subcontinuum of $2^{X}$ and $K \in \mathcal{K}$, then each component of $\bigcup \mathcal{K}$ intersects $K$.

Vol. 37, $\left.\mathrm{N}^{\circ} 1,2019\right]$ 
Proof. First, note that $\bigcup \mathcal{K} \in 2^{X}[15,2.5 .2]$. Suppose that there exists a component $C$ of $\cup \mathcal{K}$ such that $K \cap C=\emptyset$. Hence, by [4, (4.A.11)], there exist two closed subsets $K_{1}$ and $K_{2}$ of $\bigcup \mathcal{K}$ such that $\bigcup \mathcal{K}=K_{1} \cup K_{2}, K \subset K_{1}$ and $C \subset K_{2}$. Let $\mathcal{K}_{1}=\left\{L \in \mathcal{K} \mid L \subset K_{1}\right\}$ and $\mathcal{K}_{2}=\left\{L \in \mathcal{K} \mid L \cap K_{2} \neq \emptyset\right\}$. Then $\mathcal{K}_{1}$ and $\mathcal{K}_{2}$ are disjoint closed subsets of $\mathcal{K}$. Also, $\mathcal{K}=\mathcal{K}_{1} \cup \mathcal{K}_{2}$, a contradiction to the fact that $\mathcal{K}$ is connected. Therefore, each component of $\bigcup \mathcal{K}$ intersects $K$.

As a consequence of Theorem 4.12 part (1) and Lemma 4.13, we have the following corollary:

Corollary 4.14. Let $X$ be a continuum that has the property of Kelley weakly, and let $n \geq 1$. If $\mathcal{W}$ is a subcontinuum of $\mathcal{C}_{n}(X)$ such that $\operatorname{Int}_{\mathcal{C}_{n}(X)}(\mathcal{W}) \neq \emptyset$, then $\bigcup \mathcal{W} \in \mathcal{C}_{n}(X)$ and $\operatorname{Int}_{X}(\bigcup \mathcal{W}) \neq \emptyset$.

Theorem 4.15. Let $X$ be a continuum that has the property of Kelley weakly, and let $n \geq 1$. If $X$ is indecomposable, then $\mathcal{C}_{n}(X)$ is locally connected only at $X$.

Proof. Let $\left\langle U_{1}, \ldots, U_{m}\right\rangle_{n}$ be a basic open subset of $\mathcal{C}_{n}(X)$ containing $X$. Then, by [16, Theorem 2.4 and (1.4)], we have that $\left\langle U_{1}, \ldots, U_{m}\right\rangle_{n}$ is arcwise connected. Therefore, $\mathcal{C}_{n}(X)$ is locally connected at $X$.

Assume that $\mathcal{C}_{n}(X)$ is locally connected at $A$ and $A \neq X$. Then there exists a continuum neighborhood $\mathcal{K}$ of $A$ such that $\bigcup \mathcal{K} \neq X$. By part (1) of Theorem 4.12, Int $\operatorname{In}_{X}(\bigcup \mathcal{K}) \neq \emptyset$; also, by Lemma 4.13, $\bigcup \mathcal{K}$ is compact and has at most $n$ components. Hence, by the Baire Theorem [6, Theorem 2-82], some component $C$ of $\bigcup \mathcal{K}$ has nonempty interior in $X$. Therefore, since $C \neq X, X$ is decomposable by [6, Theorem 3-41].

Corollary 4.16. Let $n$ be a positive integer. If $X$ is a hereditarily indecomposable continuum, then $X$ is the only point at which $\mathcal{C}_{n}(X)$ is locally connected.

Proof. By [3, Proposition 2.7], hereditarily indecomposable continua have the property of Kelley. The corollary now follows from Theorem 4.15.

\section{References}

[1] Bellamy D.P. and Lum L., "The Cyclic Connectivity of Homogeneous Arcwise Connected Continua", Trans. Amer. Math. Soc. 266 (1981), 389-396.

[2] Camargo J., Macías S. and Uzcátegui C., "On the images of Jones' set function $\mathcal{T}$ ", Colloq. Math. 153 (2018), 1-19.

[3] Charatonik W.J., "A homogeneous continuum without the property of Kelley", Topology Appl. 96 (1999), 209-216.

[4] Christenson C. and Voxman W., Aspects of Topology, Monographs and Textbooks in Pure and Applied Math., Vol. 39, Marcel Dekker, New York, Basel, 1977.

[5] Engelking R., General Topology, Sigma series in pure mathematics, Vol. 6, Heldermann, Berlin, 1989.

[Revista Integración, temas de matemáticas 
[6] Hocking J. and Young G., Topology, Dover, 1988.

[7] Kelley J.L., "Hyprespaces of a continuum", Trans. Amer. Math. Soc. 52 (1942), $22-36$.

[8] Macías S., Topics on Continua, 2nd edition, Springer, 2018.

[9] Macías S., "A Decomposition Theorem for a Class of Continua for Which the Set Function $\mathcal{T}$ is Continuous", Colloq. Math. 109 (2007), 163-170.

[10] Macías S., "On the Idempotency of the Set Function T', Houston J. Math. 37 (2011), $1297-1305$.

[11] Macías S., "On Jones' set function $\mathcal{T}$ and the property of Kelley for Hausdorff continua", Topology Appl. 226 (2017), 51-65.

[12] Macías S., "Hausdorff continua and the uniform property of Effros", Topology Appl. 230 (2017), 338-352.

[13] Macías S. and Nadler Jr. S.B., "Various types of local connectedness in $n$-fold hyperspaces", Topology Appl. 154 (2007), 39-53.

[14] Makuchowski W., "On local connectedness in hyperspaces", Bull. Pol. Acad. Sci. 47 (1999), 119-126.

[15] Michael E., "Topologies on spaces of subsets", Trans. Amer. Math. Soc. 71 (1951), $152-182$.

[16] Misra A.K., " $C$-supersets, piecewise order-arcs and local arcwise connectedness in hyperspaces", Q. \&3 A. in General Topology, 8 (1990), 467-485.

[17] Mrówka S., "On the convergence of nets of sets", Fund. Math. 45 (1958), 237-246.

[18] Nadler Jr. S.B., Hyperspaces of Sets, Monographs and Textbooks in Pure and Applied Math., Vol. 49, Marcel Dekker, New York, Basel, 1978. Reprinted in: Aportaciones Matemáticas de la Sociedad Matemática Mexicana, Serie Textos \# 33, 2006.

[19] Wardle R.W., "On a property of J. L. Kelley", Houston J. Math. 3 (1977), 291-299.

[20] Wojdysławski M., "Sur la contractibilité des hyperspaces des continus localment connexes", Fund. Math. 30 (1938), 247-252. 